\title{
Cognitive Profile of Chronic Kidney Disease and Hemodialysis Patients without Dementia
}

\author{
James B. Post ${ }^{a, b, d} \quad$ Adejoke B. Jegede $^{b} \quad$ Kel Morin $^{b} \quad$ Ann M. Spungen ${ }^{b}$ e \\ Erik Langhoffa, d Mary Sano ${ }^{c, f}$ \\ ${ }^{a}$ Nephrology Division, ${ }^{b}$ VA RR\&D Center of Excellence for Medical Consequences of SCI, CVA RR\&D, \\ James J. Peters Veterans Affairs Medical Center, and Departments of ${ }^{\mathrm{d}}$ Medicine, ${ }^{\mathrm{e}}$ Rehabilitation Medicine, \\ and ${ }^{f}$ Psychiatry, Mount Sinai School of Medicine, Bronx, N.Y., USA
}

\section{Key Words}

Cognition · Mild cognitive impairment $\cdot$ Cerebrovascular disease $\cdot$ Chronic kidney disease $\cdot$ Hemodialysis

\begin{abstract}
Background/Aims: The high risk and prevalence of dementia among patients with chronic kidney disease (CKD) and in those receiving hemodialysis (HD) may be preceded by mild cognitive impairment $(\mathrm{MCl})$. We aimed to assess cognitive function in CKD and HD patients with no history of stroke or dementia, in order to identify and characterize early cognitive deficits. Methods: 24 CKD and 27 HD male outpatients without history of cerebrovascular or neurodegenerative disease underwent comprehensive neuropsychological testing in an observational cross-sectional study. Test results were used to categorize patients into $\mathrm{MCl}$ subtypes. Results: All subjects scored $\geq 28$ on the Mini-Mental State Examination. The prevalence of executive function was at least $25 \%$ in both groups and memory impairment occurred in $13 \%$ of the HD patients and $15 \%$ of those with CKD. MCI occurred in $76 \%$ of the group and HD patients showed a higher prevalence of $\mathrm{MCl}$ compared to CKD patients (89 vs. $63 \%)$ with a preponderance $(>70 \%)$ of cases across both groups classified as non-amnestic $\mathrm{MCl}$. Conclusion: Predi-
\end{abstract}

alysis CKD and HD patients have a high prevalence of $\mathrm{MCl}$ despite normal global cognitive function. $\mathrm{MCl}$ was more prevalent among the HD patients and deficits more frequently resulted in non-amnestic $\mathrm{MCl}$.

Copyright $\odot 2010$ S. Karger AG, Basel

\section{Introduction}

Advancing age and a heavy burden of cardiovascular risk factors among chronic kidney disease (CKD) and hemodialysis (HD) patients has focused attention on cerebrovascular morbidity in this population. Dementia prevalence in the HD population has been reported to be $30 \%$ and is frequently under recognized $[1,2]$. In patients with CKD prior to initiating dialysis, cognitive function has been correlated with the severity of renal disease and an increased risk of developing dementia [3,4]. Several factors may be responsible for the high prevalence of cognitive impairment in this population and may significantly impact cognition prior to the development of overt dementia.

Patients with CKD have a high prevalence of subcortical white matter lesions on neuroimaging and a high incidence rate for stroke $[5,6]$. White matter lesions result

\section{KARGER}

(C) 2010 S. Karger AG, Basel

Fax +41613061234 E-Mail karger@karger.ch www.karger.com www.karger.com/nec
James B. Post, MD

James J. Peters VA Medical Center, 4C-12 Outpatient Renal Practice

130 West Kingsbridge Road, Bronx, NY 10468 (USA)

Tel. +1 7185849000 , ext. 6635, Fax +1 7187414679

E-Mail James.Post@va.gov 
from subcortical small vessel disease and have been independently associated with severity of kidney disease [7]. Small vessel cerebrovascular disease leading to the development of subcortical white matter lesions is likely to be accelerated in CKD because these patients often have a clustering of traditional and emerging vascular risk factors, including hypertension, diabetes, hyperlipidemia, elevated oxidative stress, and an elevated inflammatory state. Small vessel cerebrovascular disease is the most common cause of vascular dementia with a pattern of cognitive deficits characterized by relatively preserved memory and impairment in domains related to attention, executive function and processing speed $[8,9]$. Current dementia screening tools, which rely heavily on memory deficits, may not identify patients with cognitive deficits characteristic of cerebral small vessel disease, particularly in the early stages.

Mild cognitive impairment (MCI) represents a transitional stage between cognitive changes of aging and dementia. Amnestic MCI with predominant memory deficits has gained a reputation as a predictor of incipient Alzheimer's disease [10]. Recent studies have introduced the non-amnestic MCI subtype that may serve as a potential prodrome of vascular dementia [11, 12].

The purpose of our study was to perform detailed neuropsychological testing in outpatient CKD and $\mathrm{HD}$ patients with no history of dementia, stroke or neurodegenerative disease in order to identify and characterize cognitive deficits. We hypothesized that both groups would score significantly lower when compared to normative data, particularly in domains most affected by subcortical small vessel disease. We also predicted that $\mathrm{HD}$ patients would perform worse than predialysis CKD patients due to the increased cardiovascular risk as renal disease progresses and HD is initiated.

\section{Materials and Methods}

\section{Study Design}

This is an observational, cross-sectional study of stage III-IV CKD and HD patients attending the outpatient Renal and Hemodialysis Clinics at the James J. Peters Veterans Affairs Medical Center Bronx, N.Y. Patients were recruited from October 2006 to October 2008. Outpatients were referred for screening by their primary nephrologists. All participants provided signed informed consent in accordance with local institutional review board approval. Subjects included 27 of 80 patients on HD and 24 of approximately 200 patients with stage III or stage IV CKD.

Demographic characteristics and chronic health conditions for both groups were obtained from the patient's electronic medical records. For CKD patients, the estimated glomerular filtra- tion rate (eGFR), serum calcium $(\mathrm{Ca})$, serum phosphate $\left(\mathrm{PO}_{4}\right)$, and hemoglobin levels were recorded within 3 months of neuropsychological testing. eGFR was calculated using the Modification of Diet in Renal Disease study equation [13]. Serum intact parathyroid hormone $\left(\mathrm{PTH}_{\mathrm{i}}\right)$ levels were obtained within 6 months of neuropsychological testing. When more than one value was available, averages were calculated. Laboratory values from $\mathrm{HD}$ patients included hemoglobin, $\mathrm{Ca}$ level, $\mathrm{PO}_{4}$ level, $\mathrm{PTH}_{\mathrm{i}}$ and BUN for urea reduction ratio (URR) calculation. As part of routine clinical care, these labs are drawn monthly on dialysis days prior to the treatment. Values for $\mathrm{Ca}, \mathrm{PO}_{4}, \mathrm{PTH}_{\mathrm{i}}$ and $\mathrm{URR}$ were calculated by averaging the monthly labs for 3 consecutive months prior to neuropsychological testing. All tests were performed at a single central laboratory using standard methods. Labs were drawn for thyroid-stimulating hormone (TSH) within 1 week of neuropsychological testing and values determined by radioimmunoassay for both groups. Measured blood pressure was determined by averaging the 3 clinical appointment blood pressure readings prior to neuropsychological testing.

\section{Participants}

Patients were considered for enrollment if they were fluent in English and able to complete baseline assessments. HD participants had to be receiving treatment for at least 3 months and have a 3 -month average URR of $\geq 65 \%$ at the time of screening. Those receiving HD were dialyzed 3 days per week for 3-4 hours per session. Exclusion criteria were as follows: a history of stroke or dementia when questioned or if documented in the medical chart, a history of Parkinson's or neurodegenerative disease, liver function enzymes (AST and ALT) more than two times the upper limit of normal, or a hemoglobin level $<10 \mathrm{~g}$. Stage III CKD was defined as an eGFR $30-59 \mathrm{ml} / \mathrm{min} / 1.73 \mathrm{~m}^{2}$ and stage IV CKD was defined as eGFR $15-29 \mathrm{ml} / \mathrm{min} / 1.73 \mathrm{~m}^{2}$. All the subjects that completed testing were male. All attempts were made to perform neuropsychological testing on non-dialysis days in order to avoid the potential temporal relationship between cognitive function and time since last dialysis. However, 5 patients were tested on dialysis days due to scheduling difficulties.

\section{Measurements}

A 60-min battery of nine validated neuropsychological tests was administered to all study participants under supervision of a senior psychologist with specialized training in neuropsychology. The neurocognitive battery included: The Mini-Mental State Examination (MMSE) [14], The California Verbal Learning Test-II (CVLT-II) Standard Form [15], the Controlled Oral Word Association Test (COWAT-FAS) [16], animal category fluency, Digit Span [17], the Symbol Digit Modality Test (SDMT) [18], Trails A \& B [19], Stroop Word \& Color tests [20], and the Short Category Test (SCT) [21] (table 1).

Neuropsychological Test Analysis and MCI Classification

In the absence of a matched control group, we compared group raw scores on each cognitive test to previously published normative data on age- and education-matched healthy controls. For between-group comparisons on each cognitive measure, raw test scores were converted to the published age- and education-standardized $\mathrm{T}$ scores. $\mathrm{T}$ scores that were $\geq 1.5$ standard deviations (SD) below the mean were identified as impaired. To calculate the domain composite $\mathrm{T}$ scores, we first calculated a mean $\mathrm{T}$ score of 
Table 1. Neurocognitive battery

\begin{tabular}{ll}
\hline Test & Function assessed \\
\hline Mini-Mental State Examination & Global cognitive status \\
Attention and processing speed & Visual attention \\
Trails A & Auditory attention and memory \\
Digit Span & Visuomotor scanning and processing \\
Symbol Digit Modalities Test & Speed of information processing \\
Stroop Word & Speed of information processing \\
Stroop Color & \\
Executive function & Concept formation and reasoning \\
Short Category Test & Set shifting \\
Trails B & Cognitive flexibility \\
Stroop Color-Word & \\
Language & Verbal fluency, phonemic naming \\
Controlled Oral Word Association Test (FAS) & Verbal fluency, categorical naming \\
Animal naming & \\
Memory & Verbal immediate, short-term, long-term \\
California Verbal Learning Test-II & and delayed recognition memory \\
\hline
\end{tabular}

all the completed cognitive tests within each particular domain (table 1) for each subject. Next, all subjects' domain composite scores were averaged within each group to calculate the overall domain composite score for either CKD or HD groups. Results of neurocognitive tests were used to classify subjects with an MCI subtype by using diagnostic criteria utilized from the Cardiovascular Health Cognition Study and the Mayo Clinic Study of Aging as approximate guidelines $[12,22,23]$. Subjects with a composite score for long and short delayed memory tests $\geq 1.5$ SD below ageand education-matched norms were classified as MCI amnestic type. Study participants who did not meet criteria for MCI amnestic type, but scored $\geq 1.5$ SD below the norm on two or more tests in a non-memory domain (Attention/Processing Speed, Executive Function, or Language) were classified as MCI non-amnestic type. The MCI amnestic subtype was further categorized into single domain involvement if the memory domain was only impaired and multiple domain if the subject scored $\geq 1.5$ SD below the norm on two or more tests within a non-memory domain. Subjects with non-amnestic MCI were further characterized into single domain if only one non-memory domain was impaired or multiple domain if the subject scored $\geq 1.5$ SD below the norm on one or more tests in an additional non-memory domain.

\section{Statistical Methods}

Descriptive statistics are reported as means and SDs or percent as appropriate. Within each study group, single group t-tests were used to determine raw score differences between the CKD study participants and previously published normative (PN) data for age-matched, healthy controls. Multiple regression models were used to adjust for differences between the CKD and HD groups for diabetes mellitus (DM), hypertension (HTN), hyperlipidemia, coronary artery disease (CAD), congestive heart failure $(\mathrm{CHF})$, peripheral arterial disease (PAD), $\mathrm{PO}_{4}, \mathrm{Ca} \times \mathrm{PO}_{4}, \mathrm{PTH}_{\mathrm{i}}$ and $\mathrm{TSH}$. Independent sample t-tests were used to evaluate differenc- es between the $\mathrm{CKD}$ and $\mathrm{HD}$ groups on individual cognitive test $\mathrm{T}$ scores and domain composite $\mathrm{T}$ scores. CKD and HD groups were compared on the demographic and clinical variables represented as a continuous number by using t-tests. For the dichotomous or categorical variables, $\chi^{2}$ tests were used to determine the significance of differences in prevalence between the groups. The statistical analyses were performed using StatView (SAS, 1998). Analysis of covariance (ANCOVA) was used to determine the differences between those with and without MCI for traditional and emerging cardiovascular risk factors.

In the CKD group, visual difficulties prevented 2 subjects from completing two or more of the following: Trails A, SDMT, Digit Span, Stroop Word, Stroop Color, Stroop Color-Word, and Trails B. Six patients refused to complete the Short Category Test out of frustration. In the HD group, 1 subject did not complete Stroop Color and Stroop Color-Word secondary to color vision deficiency. Scores from individual neurocognitive measures that were not completed were excluded from data analysis and not counted.

\section{Results}

\section{Participants}

Fifty-one male subjects participated in the study, 24 with CKD and 27 on $\mathrm{HD}$. CKD subjects were significantly older than those receiving $\mathrm{HD}(72 \pm 12$ vs. $63 \pm 11$ years, $\mathrm{p}=0.01$ ). No other demographic variables were statistically different between the groups (table 2). Hypertension, diabetes, and hyperlipidemia were highly prevalent in both the CKD and HD groups, with no significant 
Table 2. Characteristics of study participants

\begin{tabular}{|c|c|c|c|}
\hline & $\begin{array}{l}\text { CKD } \\
(\min -\max )\end{array}$ & $\begin{array}{l}\text { HD } \\
(\min -\max )\end{array}$ & $\mathrm{p}$ \\
\hline Age, years & $72 \pm 12(39-87)$ & $63 \pm 11(45-82)$ & 0.01 \\
\hline Education, years & $13 \pm 4(2-21)$ & $13 \pm 1(11-16)$ & 0.8 \\
\hline Years on dialysis & N/A & $2.2 \pm 2.3(0.3-10)$ & \\
\hline URR, \% & N/A & $71 \pm 4(65-82)$ & \\
\hline \multicolumn{4}{|l|}{ CKD status } \\
\hline Stage III & $16(67)$ & N/A & \\
\hline Stage IV & $8(33)$ & N/A & \\
\hline Stage V & N/A & $27(100)$ & \\
\hline \multicolumn{3}{|l|}{ Ethnicity } & 0.2 \\
\hline African-American & $10(42)$ & $17(63)$ & \\
\hline Caucasian & $9(38)$ & $5(19)$ & \\
\hline Other & $5(21)$ & $5(19)$ & \\
\hline \multicolumn{4}{|c|}{ Traditional cardiovascular risk factors } \\
\hline Hypertension & $24(100)$ & $24(89)$ & 0.2 \\
\hline Diabetes & $16(67)$ & $14(52)$ & 0.4 \\
\hline Hyperlipidemia & $6(25)$ & $5(19)$ & 0.7 \\
\hline \multicolumn{4}{|l|}{ Systolic BP } \\
\hline $\mathrm{mm} \mathrm{Hg}$ & $142 \pm 16$ & $145 \pm 17$ & 0.6 \\
\hline \multicolumn{4}{|l|}{ Diastolic BP } \\
\hline $\mathrm{mm} \mathrm{Hg}$ & $74 \pm 15$ & $77 \pm 13$ & 0.4 \\
\hline \multicolumn{4}{|c|}{ Emerging cardiovascular risk factors } \\
\hline $\mathrm{PO}_{4}, \mathrm{mg} / \mathrm{dl}$ & $3.8 \pm 0.8$ & $5.4 \pm 1.0$ & $<0.001$ \\
\hline $\mathrm{Ca} \times \mathrm{PO}_{4}$ & $33.4 \pm 6.7$ & $48.0 \pm 11.0$ & $<0.001$ \\
\hline $\mathrm{PTH}_{\mathrm{i}}, \mathrm{ng} / \mathrm{l}$ & $128.1 \pm 80.8$ & $345.9 \pm 269.7$ & $<0.001$ \\
\hline
\end{tabular}

$\mathrm{HD}=$ Hemodialysis CKD = chronic kidney disease (stage IIIIV); URR = urea reduction ratio; $\mathrm{PO}_{4}=$ serum phosphate; $\mathrm{Ca} \times$ $\mathrm{PO}_{4}=$ calcium phosphate product; $\mathrm{PTH}_{\mathrm{i}}=$ parathyroid hormone (intact).

difference in proportion of these factors between groups. The HD group had significantly higher $\mathrm{PO}_{4}, \mathrm{Ca} \times \mathrm{PO}_{4}$ product, and $\mathrm{PTH}_{\mathrm{i}}$. There were no differences between the groups for blood pressure (table 2). Overall, the HD group was adequately dialyzed, with an average 3 -month URR of $71 \pm 4 \%$ and no subject having less than $65 \%$. Both groups had a prevalence of coronary artery disease (58\% CKD , 37\% HD), congestive heart failure (33\% CKD, $22 \% \mathrm{HD}$ ) and peripheral arterial disease (21\% CKD, $19 \%$ $\mathrm{HD})$. There was no significant difference in prevalence between groups for CAD, CHF, and PAD. Hemoglobin levels were significantly different between $\mathrm{CKD}$ and $\mathrm{HD}$ groups (12.1 \pm 1.6 vs. $11.4 \pm 1.0, p=0.04$, respectively), and none were $<10 \mathrm{~g}$ as per the exclusion criterion. Hemoglobin level was not found to significantly affect cognitive performance on the given tasks. The CKD group had a significantly higher TSH level than the HD group $(2.3 \pm 2.1$ vs. $1.4 \pm 0.8, \mathrm{p}=0.05)$.

\section{Raw Scores versus Published Norms}

The raw scores compared with the published norms (PN) for the CKD and HD groups are presented (table 3). All subjects scored $\geq 28$ on MMSE. In the CKD group, subjects scored significantly lower on 4 of 5 measures of attention and processing speed. In the domain of executive function, CKD subjects scored significantly lower than published norms on all measures. In the CKD group, only 1 of 4 measures of memory was significantly lower than the published norms. In the HD group, subjects scored significantly lower on all measures for each cognitive domain versus the published norms.

\section{T Scores for Individual Tests: CKD versus HD}

In order to compare groups, T scores for each neuropsychological test were calculated and reported (table 4). HD subjects scored significantly lower than CKD subjects on 3 of 5 measures of attention and processing speed and all measures of language. CKD subjects scored significantly lower than HD subjects on one test of executive function. There was no significant difference between the groups for measures of memory. When the T scores were adjusted for hypertension, diabetes, hyperlipidemia, CAD, CHF, PAD, $\mathrm{PO}_{4}, \mathrm{Ca} \times \mathrm{PO}_{4}, \mathrm{PTH}_{\mathrm{i}}$ and TSH, significant differences between the groups remained (data not shown). For the HD group, time since the last dialysis was not significantly associated with performance on the cognitive tests.

\section{Composite T Scores and Prevalence of Impairment by \\ Domain: CKD versus $H D$}

HD subjects had significantly lower composite $\mathrm{T}$ scores in the domains of attention and processing speed and language ( $34 \pm 5$ vs. $40 \pm 7, \mathrm{p}<0.01$, and $39 \pm 7 \mathrm{vs.}$ $47 \pm 9, \mathrm{p}<0.01$, respectively). The proportion of subjects with attention and processing speed impairment was significantly greater in the HD compared with the CKD group ( 59 vs. $25 \%, \mathrm{p}=0.01$ ). The prevalence of executive function impairment was high in both groups (30 and 25\%). Memory impairment was least prevalent among HD patients and present in $13 \%$ of those with CKD (table 4).

\section{Prevalence and Subtype of MCI}

A flow chart of the prevalence and subtype of $\mathrm{MCI}$ is presented (fig. 1). Of those subjects with MCI, 26\% (10 of 39) met the criteria for amnestic MCI and all but 1 of 
Table 3. Raw scores on cognitive function tests versus published norms

\begin{tabular}{|c|c|c|c|c|c|c|}
\hline \multirow[t]{2}{*}{ Test } & \multicolumn{3}{|l|}{ CKD } & \multicolumn{3}{|l|}{ HD } \\
\hline & raw & $\mathrm{PN}$ & $\mathrm{p}$ & raw & $\mathrm{PN}$ & $\mathrm{p}$ \\
\hline MMSE & $28.9 \pm 1.2$ & & & $28.1 \pm 1.8$ & & \\
\hline \multicolumn{7}{|l|}{ Attention and processing speed } \\
\hline Trails A & $59.5 \pm 24.2$ & $47.2 \pm 11.6$ & 0.03 & $64.7 \pm 24.8$ & $39.5 \pm 8.8$ & $<0.001$ \\
\hline Digit Span & $12.9 \pm 2.4$ & $15.2 \pm 0.8$ & $<0.001$ & $12.4 \pm 3.3$ & $15.9 \pm 0.8$ & $<0.001$ \\
\hline SDMT & $29.8 \pm 8.7$ & $32.5 \pm 9.5$ & 0.3 & $29.1 \pm 7.6$ & $41.1 \pm 10.1$ & $<0.001$ \\
\hline Stroop Word & $78.1 \pm 13.5$ & $97.6 \pm 8.6$ & $<0.001$ & $68.4 \pm 11.7$ & $99.1 \pm 2.7$ & $<0.001$ \\
\hline Stroop Color & $45.8 \pm 8.1$ & $71.5 \pm 4.8$ & $<0.001$ & $49.9 \pm 11.6$ & $73.1 \pm 2.2$ & $<0.001$ \\
\hline \multicolumn{7}{|l|}{ Executive function } \\
\hline Short Category Test & $43.4 \pm 8.3$ & $31.4 \pm 2.9$ & $<0.001$ & $38.3 \pm 13.8$ & $31.5 \pm 2.7$ & 0.02 \\
\hline Trails B & $157.5 \pm 56.8$ & $115.6 \pm 33.0$ & 0.005 & $169.6 \pm 93.6$ & $92.8 \pm 26.8$ & $<0.001$ \\
\hline Stroop Color-Word & $20.6 \pm 4.4$ & $32.9 \pm 6.5$ & $<0.001$ & $21.4 \pm 7.7$ & $35.1 \pm 3.2$ & $<0.001$ \\
\hline \multicolumn{7}{|l|}{ Language } \\
\hline FAS & $30.3 \pm 12.1$ & $35.9 \pm 6.1$ & 0.05 & $25.4 \pm 9.9$ & $39.6 \pm 4.2$ & $<0.001$ \\
\hline Animal naming & $15.6 \pm 4.0$ & $16.8 \pm 2.2$ & 0.2 & $13.2 \pm 4.2$ & $18.3 \pm 2.2$ & $<0.001$ \\
\hline \multicolumn{7}{|l|}{ Memory } \\
\hline CVLT total recall & $34.5 \pm 8.5$ & $36.4 \pm 6.0$ & 0.4 & $33.7 \pm 7.4$ & $40.5 \pm 5.8$ & $<0.001$ \\
\hline CVLT short delay recall & $6.1 \pm 3.2$ & $7.4 \pm 1.4$ & 0.08 & $5.9 \pm 3.7$ & $8.1 \pm 1.1$ & 0.006 \\
\hline CVLT long delay recall & $6.7 \pm 2.8$ & $7.8 \pm 1.3$ & 0.09 & $6.6 \pm 3.3$ & $8.5 \pm 1.0$ & 0.008 \\
\hline CVLT long delay recognition & $12.5 \pm 2.5$ & $14.0 \pm 0.2$ & 0.007 & $12.3 \pm 2.3$ & $14.0 \pm 0.0$ & $<0.001$ \\
\hline
\end{tabular}

$\mathrm{HD}=$ Hemodialysis; $\mathrm{CKD}=$ chronic kidney disease $\mathrm{PN}=$ published normative value.

Scores are reported as mean $\pm \mathrm{SD}$.

Trails A, SDMT, Digit Span, STROOP Word $\mathrm{n}=23$ CKD, $\mathrm{n}=27$ HD; SCT $\mathrm{n}=18$ CKD, $\mathrm{n}=27$ HD.

STROOP Color, STROOP Color-Word $n=22$ CKD, $n=26$ HD; Trails B $n=22$ CKD, $n=27$ HD.

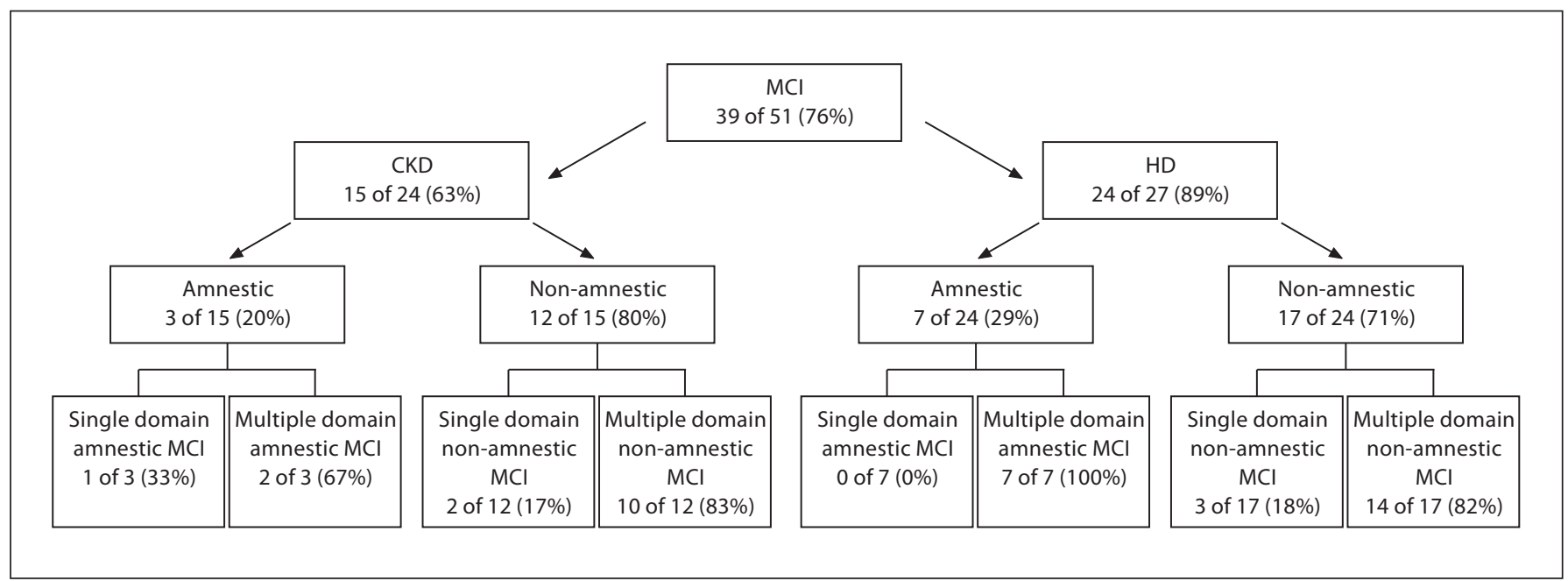

Fig. 1. Mild cognitive impairment by subtype. Diagnostic criteria: Amnestic MCI single domain was defined as $\geq 1.5$ SD below the norm on delayed recall composite score. Amnestic MCI multiple domain was defined as $\geq 1.5 \mathrm{SD}$ below the norm on delayed recall composite score and 2 tests $\geq 1.5$ SD below the norm in any one domain other than memory. Non-amnestic MCI single domain was defined as 2 tests $\geq 1.5 \mathrm{SD}$ below the norm in any one domain other than memory. Non-amnestic MCI single domain was defined as 2 tests $\geq 1.5$ SD below the norm in any one domain other than memory and 1 test $\geq 1.5$ SD below the norm in an additional domain other than memory. 
Table 4. Cognitive function scores and prevalence of impairment by group

\begin{tabular}{|c|c|c|c|c|c|c|}
\hline \multirow[t]{2}{*}{ Test } & \multicolumn{3}{|l|}{ T score } & \multicolumn{3}{|c|}{ Impaired } \\
\hline & CKD & $\mathrm{HD}$ & $\mathrm{p}$ & CKD & HD & $\mathrm{p}$ \\
\hline Attention and processing speed & $40.0 \pm 7.1$ & $34.0 \pm 5.2$ & 0.001 & $6(25)$ & $16(59)$ & 0.02 \\
\hline Trails $\mathrm{A}^{\mathrm{a}}$ & $41.8 \pm 16.8$ & $32.1 \pm 11.7$ & 0.02 & & & \\
\hline Digit Span $^{\mathrm{a}}$ & $45.0 \pm 6.4$ & $42.5 \pm 7.4$ & 0.2 & & & \\
\hline $\mathrm{SDMT}^{\mathrm{b}}$ & $46.4 \pm 11.6$ & $36.0 \pm 10.2$ & 0.001 & & & \\
\hline Stroop Word ${ }^{b}$ & $37.0 \pm 9.8$ & $28.3 \pm 7.6$ & 0.001 & & & \\
\hline Stroop Color ${ }^{\mathrm{b}}$ & $29.1 \pm 7.7$ & $31.0 \pm 8.9$ & 0.4 & & & \\
\hline Executive function & $40.2 \pm 7.7$ & $38.9 \pm 7.3$ & 0.5 & $6(25)$ & $8(30)$ & 0.8 \\
\hline Short Category Test ${ }^{\mathrm{a}}$ & $39.9 \pm 7.0$ & $46.5 \pm 12.6$ & 0.05 & & & \\
\hline Trails B ${ }^{\mathrm{a}}$ & $40.2 \pm 15.7$ & $33.0 \pm 13.7$ & 0.09 & & & \\
\hline Stroop Color-Word ${ }^{b}$ & $38.5 \pm 6.7$ & $36.8 \pm 7.3$ & 0.4 & & & \\
\hline Language $^{\mathrm{b}}$ & $46.6 \pm 8.8$ & $38.9 \pm 6.7$ & $<0.001$ & $2(8)$ & $8(30)$ & 0.08 \\
\hline FAS & $45.1 \pm 11.0$ & $38.9 \pm 8.2$ & 0.03 & & & \\
\hline Animal naming & $48.3 \pm 11.0$ & $38.9 \pm 10.9$ & 0.004 & & & \\
\hline Memory $^{\mathrm{a}}$ & $45.7 \pm 9.1$ & $42.7 \pm 8.2$ & 0.2 & $3(13)$ & $4(15)$ & 0.9 \\
\hline CVLT total recall & $48.3 \pm 10.6$ & $43.2 \pm 8.2$ & 0.06 & & & \\
\hline CVLT short delay recall & $46.7 \pm 11.5$ & $42.8 \pm 11.3$ & 0.2 & & & \\
\hline CVLT long delay recall & $46.9 \pm 10.4$ & $44.7 \pm 9.6$ & 0.4 & & & \\
\hline CVLT long delay recognition & $40.8 \pm 13.2$ & $39.8 \pm 12.4$ & 0.8 & & & \\
\hline 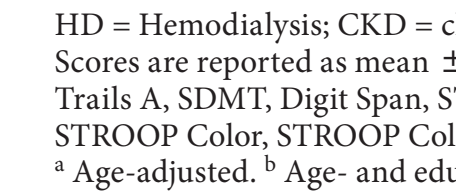 & $\begin{array}{l}\text { hey disease }(\mathrm{s} \\
\text { irment recor } \\
\text { ord } \mathrm{n}=23 \mathrm{Cl} \\
=22 \mathrm{CKD}, \mathrm{n} \\
\text { usted ( } \mathrm{T} \text { scor }\end{array}$ & $\begin{array}{l}\text { III-IV). } \\
\text { s n (\%). } \\
=27 \text { HD; SC } \\
\text { HD; Trails B }\end{array}$ & $\begin{array}{l}=18 \mathrm{CK} \\
22 \mathrm{CKD}\end{array}$ & $\begin{array}{l}\text { HD. } \\
\text { D. }\end{array}$ & & \\
\hline
\end{tabular}

these had multiple domain involvement. The remaining $74 \%$ (29 of 39) were characterized as having non-amnestic MCI, of whom $83 \%$ (24 of 29 ) had multiple domain involvement.

\section{Traditional and Emerging Cardiovascular Risk}

Factors: $M C I$ versus Non-MCI

No significant differences were found between the groups with $\mathrm{MCI}$ and without MCI or between MCI subtypes for $\mathrm{PO}_{4}$ level, $\mathrm{Ca} \times \mathrm{PO}_{4}$ product, $\mathrm{PTH}_{\mathrm{i}}$ level, TSH level, measured blood pressure or history of hypertension, diabetes, and hyperlipidemia, CAD, CHF, and PAD (data not shown).

\section{Discussion}

In our CKD and HD outpatients with normal global cognitive function and no history of dementia, stroke or neurodegenerative disease, a high prevalence of neuropsychological deficits was detected compared to norma- tive samples. Examination of clinically meaningful deficits with significant impairment meeting the criteria for MCI revealed that these deficits were most common in non-memory domains. These results are supported by an earlier study of HD patients without a clinical history of dementia or stroke where cognitive dysfunction was primarily subcortical in nature with a relative sparing of cortical domain function [24].

MCI is a transitional state between normal cognition and the earliest clinical features of dementia. Amnestic and non-amnestic subtypes have been identified based on specific domain involvement with each subtype influencing the subsequent development of Alzheimer's or vascular dementia [25]. For example, amnestic MCI has been established as a prodrome of Alzheimer's disease, whereas non-amnestic MCI more frequently predicts the development of vascular dementia.

A high prevalence of MCI was identified in the overall group and among HD patients versus CKD patients with a preponderance of cases classified as non-amnestic MCI. It is noteworthy that even among the amnestic MCI cases, 
all but 1 subject also had non-memory deficits. In fact, most of our MCI cases had multiple non-amnestic domain involvement, which has been shown to increase the risk of developing vascular dementia and increase the risk of mortality versus amnestic MCI [25]. Although all patients had a normal MMSE, their profile of neuropsychological deficits are likely to affect planning, sequencing, organization, and mental flexibility all of which are required for independence in activities of daily living and compliance with complex medical regimens. Because subcortical vascular dementia secondary to cerebral small vessel disease has a relatively insidious onset with gradual cognitive decline often associated with nonmemory domains, relying on routine neuropsychological instruments that require memory deficit for diagnosis could underdiagnose non-amnestic deficits, increasing the renal patient's risk for treatment non-compliance and failure. Early detection of non-amnestic deficits characteristic of cerebral small vessel disease may provide an opportunity for interventions aimed to slow disease progression, such as more vigorous control of blood pressure, dyslipidemia, and other metabolic perturbations as well as cognitive therapy. Future trials should then be designed to evaluate the efficacy of interventions to slow the progression of MCI to overt dementia.

When compared to CKD patients, our HD patients scored significantly lower on several measures of attention and mental processing speed and language with no significant change on any measure of memory between groups. Impairments in the domains of attention and processing speed, executive function, and language with a relative preservation of memory is reminiscent of the cognitive changes associated with the early manifestations of vascular dementia secondary to small vessel cerebrovascular disease [26]. This pattern of cognitive deficit has been associated with manifestations of cerebral small vessel disease such as white matter lesions [27-31]. A potential link between kidney disease and subcortical cognitive deficits comes from evidence that albuminuria has been associated with executive function deficits and volume of white matter hyperintensities [32]. Subcortical white matter lesions are highly prevalent in advanced CKD and associated with renal disease severity [7]. Significantly lower measures of subcortical cognitive function in the HD group versus the CKD group, despite comparable memory scores, suggests that worsening cognitive deficits related to small vessel disease may parallel the progression of renal disease.

In our patients, the limited number of traditional and emerging cardiovascular risk factors identified was not found to be associated with the lower cognitive scores in the HD group compared with the CKD group. These cardiovascular risk factors were equally prevalent between patients with and without MCI. However, mechanisms for cognitive deficits characteristic of cerebral small vessel disease among CKD and HD patients may involve the cumulative effect of multiple vascular risk factors. As renal function declines, levels of nitric oxide synthase inhibitor asymmetric dimethyl-L-arginine increase, suppressing the synthesis of nitric oxide [33]. Nitric oxide is an inhibitor of vascular smooth muscle cell proliferation, platelet aggregation, and a potent vasodilator. Endothelial dysfunction resulting from reduced nitric oxide production in cerebral small vessels may contribute to the development of chronic ischemic damage of subcortical structures. Endothelial dysfunction has been associated with white matter hyperintensities in older adults and with a decrease in vasodilatory capacity of the cerebral cortex [34]. Cerebral small vessel damage is likely to be exacerbated in patients with advancing CKD through increased levels of inflammation [35], advanced glycation end products [36, 37], and oxidant stress [38] that occur as renal function deteriorates. In patients receiving $\mathrm{HD}$, frequent and repetitive hypotensive episodes during treatment may cause further damage to ischemia-sensitive frontal-subcortical circuits because small vessel arteriosclerosis, calcification, and nitric oxide deficiency may likely interfere with normal mechanisms of autoregulation and preservation of blood flow to these important anterior cerebral structures. The significantly decreased carotid blood flow that appears to improve following a single dialysis treatment and the decreased cerebral oxygenation that has been reported in HD patients are not yet fully understood [39]. In patients with advancing kidney disease, cumulative small vessel damage with subsequent damage to subcortical white matter structures may lead to early subcortical cognitive deficits. Even though our patients had no clinical history of stroke, significant underlying cerebrovascular disease cannot be excluded since neuroimaging was not available. The observed nonamnestic deficits may be an indicator of underlying cerebrovascular damage since kidney disease has been shown to independently predict subclinical brain infarcts [40]. As renal function deteriorates and patients require HD, the cognitive deficits are likely to progress and ultimately result in overt vascular dementia.

The strength of our study is that a relatively healthy group of CKD and HD patients with no clinical history of dementia, stroke or neurodegenerative disease was evaluated, allowing us to identify a profile of cognitive 
deficits that may be helpful in determining the future progression to dementia. We also required that all patients had hemoglobin levels $>10 \mathrm{~g}$, normal liver function tests, and adequate dialysis in order to eliminate these confounders as causes of cognitive impairment. Nevertheless, our study had a relatively small number of subjects and other limitations. For example, while stroke was excluded by history and medical record review, imaging was not available. In addition, all of our subjects were male which limits generalization of our findings to female patients. Another possible limitation may have been in the diagnosis of MCI based on neurocognitive scores in the absence of a subjective clinical complaint of memory or cognitive decline.

In conclusion, we found evidence of significant cognitive impairment in CKD and HD outpatients with nor- mal global cognitive function and no history of stroke or neurodegenerative diseases. These impairments were predominantly in non-memory domains, thus indicating a potential vascular etiology. In patients with advanced CKD, amnestic MCI may precede overt dementia and be underdiagnosed with routine screening measures such as the MMSE. Early recognition of MCI prior to the development of overt dementia may provide an opportunity to intervene and slow the progression of disease.

\section{Acknowledgements}

Funding for this project was provided by the VA VISN 3 Seed Grant Program, VARR\&D CDA 2 \#B5050W, VARR\&D Center of Excellence \#B4162C and NIH/NIA \#P50AG005138.

\section{References}

1 Sehgal AR, Grey SF, DeOreo PB, Whitehouse PJ: Prevalence, recognition, and implications of mental impairment among hemodialysis patients. Am J Kidney Dis 1997;30:4149.

-2 Murray AM, Tupper DE, Knopman DS, Gilbertson DT, Pederson SL, Li S, Smith GE, Hochhalter AK, Collins AJ, Kane RL: Cognitive impairment in hemodialysis patients is common. Neurology 2006;67:216-223.

3 Kurella Tamura M, Wadley V, Yaffe K, McClure LA, Howard G, Go R, Allman RM, Warnock DG, McClellan W: Kidney function and cognitive impairment in us adults: the Reasons for Geographic and Racial Differences in Stroke (REGARDS) Study. Am J Kidney Dis 2008;52:227-234.

4 Kurella M, Chertow GM, Luan J, Yaffe K: Cognitive impairment in chronic kidney disease. J Am Geriatr Soc 2004;52:18631869.

5 Martinez-Vea A, Salvado E, Bardaji A, Gutierrez C, Ramos A, Garcia C, Compte T, Peralta C, Broch M, Pastor R, Angelet P, Marcas L, Sauri A, Oliver JA: Silent cerebral white matter lesions and their relationship with vascular risk factors in middle-aged predialysis patients with CKD. Am J Kidney Dis 2006; $47: 241-250$

-6 Fazekas G, Fazekas F, Schmidt R, Kapeller P, Offenbacher H, Krejs GJ: Brain MRI findings and cognitive impairment in patients undergoing chronic hemodialysis treatment. J Neurol Sci 1995; 134:83-88.

7 Ikram MA, Vernooij MW, Hofman A, Niessen WJ, van der Lugt A, Breteler MM: Kidney function is related to cerebral small vessel disease. Stroke 2008;39:55-61.
8 Looi JC, Sachdev PS: Differentiation of vascular dementia from AD on neuropsychological tests. Neurology 1999;53:670-678.

-9 Roman GC, Royall DR: Executive control function: a rational basis for the diagnosis of vascular dementia. Alzheimer Dis Assoc Disord 1999;13(suppl 3):S69-S80.

10 Petersen RC, Negash S: Mild cognitive impairment: an overview. CNS Spectr 2008; 13 : 45-53.

11 Lopez OL, Jagust WJ, DeKosky ST, Becker JT, Fitzpatrick A, Dulberg C, Breitner J, Lyketsos C, Jones B, Kawas C, Carlson M, Kuller LH: Prevalence and classification of mild cognitive impairment in the Cardiovascular Health Study Cognition Study: part 1. Arch Neurol 2003;60:1385-1389.

12 Roberts RO, Geda YE, Knopman DS, Cha RH, Pankratz VS, Boeve BF, Ivnik RJ, Tangalos EG, Petersen RC, Rocca WA: The Mayo Clinic Study of Aging: design and sampling, participation, baseline measures and sample characteristics. Neuroepidemiology 2008; 30:58-69.

13 K/DOQI Clinical Practice Guidelines for Chronic Kidney Disease: Evaluation, classification, and stratification. Am J Kidney Dis 2002;39:S1-S266.

14 Folstein MF FS, McHugh PR: Mini-Mental State: a practical method for grading the cognitive state of patients for the clinician. J Psychiatr Res 1975;12:189-198.

15 Delis D, Kramer J, Kaplan E, Ober B: California Verbal Learning Test, ed 2 (CVLT-II). San Antonio, Psychological Corp, 2000.

16 Benton AL, Hamsher KD: Multilingual Aphasia Examination, ed 3. Iowa City, AJA Associates, 1994.
17 Wechsler D: Wechsler Memory Scale, ed 3. Administration and Scoring Manual. San Antonio, Psychological Corp, 1997.

18 Smith A: Symbol Digit Modality Test (SDMT). Los Angeles, Western Psychological Services, 1991.

19 Reitan RWD: The Halstead-Reitan Neuropsychological Test Battery. Tuscon, Neuropsychology Press, 1988.

20 Golden JC: Stroop Color and Word Test. Chicago, Stoeling Corp, 1978.

21 Wetzel L, Boll T: Short Category Test, Booklet Format. Los Angeles, Western Psychological Services, 1986.

22 Lopez OL, Kuller LH, Fitzpatrick A, Ives D, Becker JT, Beauchamp N: Evaluation of dementia in the Cardiovascular Health Cognition Study. Neuroepidemiology 2003;22:112.

23 Petersen RC: Mild cognitive impairment as a diagnostic entity. J Intern Med 2004;256: 183-194.

-24 Pereira AA, Weiner DE, Scott T, Chandra P, Bluestein R, Griffith J, Sarnak MJ: Subcortical cognitive impairment in dialysis patients. Hemodial Int 2007;11:309-314.

$\longrightarrow 25$ Yaffe K, Petersen RC, Lindquist K, Kramer J, Miller B: Subtype of mild cognitive impairment and progression to dementia and death. Dement Geriatr Cogn Disord 2006; 22:312-319.

-26 Pugh KG, Lipsitz LA: The microvascular frontal-subcortical syndrome of aging. Neurobiol Aging 2002;23:421-431.

$\longrightarrow 27$ De Groot JC, de Leeuw FE, Oudkerk M, van Gijn J, Hofman A, Jolles J, Breteler MM: Cerebral white matter lesions and cognitive function: The Rotterdam Scan Study. Ann Neurol 2000;47:145-151. 
-28 Ylikoski R, Ylikoski A, Erkinjuntti T, Sulkava $R$, Raininko R, Tilvis R: White matter changes in healthy elderly persons correlate with attention and speed of mental processing. Arch Neurol 1993;50:818-824.

-29 Almkvist O, Wahlund LO, Andersson-Lundman G, Basun H, Backman L: White-matter hyperintensity and neuropsychological functions in dementia and healthy aging. Arch Neurol 1992;49:626-632.

>30 Boone KB, Miller BL, Lesser IM, Mehringer CM, Hill-Gutierrez E, Goldberg MA, Berman NG: Neuropsychological correlates of white-matter lesions in healthy elderly subjects. A threshold effect. Arch Neurol 1992; 49:549-554.

-31 Meyer JS, Quach M, Thornby J, Chowdhury M, Huang J: MRI identifies MCI subtypes: vascular versus neurodegenerative. J Neurol Sci 2005;230:121-129.
32 Weiner DE, Bartolomei K, Scott T, Price LL, Griffith JL, Rosenberg I, Levey AS, Folstein MF, Sarnak MJ: Albuminuria, cognitive functioning, and white matter hyperintensities in homebound elders. Am J Kidney Dis 2009;53:438-447.

33 Fleck C, Janz A, Schweitzer F, Karge E, Schwertfeger M, Stein G: Serum concentrations of asymmetric (ADMA) and symmetric (SDMA) dimethylarginine in renal failure patients. Kidney Int Suppl 2001; 78:S14-S18.

34 Hoth KF, Tate DF, Poppas A, Forman DE, Gunstad J, Moser DJ, Paul RH, Jefferson AL, Haley AP, Cohen RA: Endothelial function and white matter hyperintensities in older adults with cardiovascular disease. Stroke 2007;38:308-312.

35 Arici M, Walls J: End-stage renal disease, atherosclerosis, and cardiovascular mortality: is C-reactive protein the missing link? Kidney Int 2001;59:407-414.

\$6 Weiss MF, Erhard P, Kader-Attia FA, Wu YC, Deoreo PB, Araki A, Glomb MA, Monnier VM: Mechanisms for the formation of glycoxidation products in end-stage renal disease. Kidney Int 2000;57:2571-2585.
37 Raj DS, Choudhury D, Welbourne TC, Levi M: Advanced glycation end products: a nephrologist's perspective. Am J Kidney Dis 2000;35:365-380.

38 Himmelfarb J, Stenvinkel P, Ikizler TA, Hakim RM: The elephant in uremia: oxidant stress as a unifying concept of cardiovascular disease in uremia. Kidney Int 2002;62: 1524-1538.

39 Prohovnik I, Post J, Uribarri J, Lee H, Sandu O, Langhoff E: Cerebrovascular effects of hemodialysis in chronic kidney disease. J Cereb Blood Flow Metab 2007;27:1861-1869.

40 Seliger SL, Longstreth WT Jr, Katz R, Manolio T, Fried LF, Shlipak M, Stehman-Breen CO, Newman A, Sarnak M, Gillen DL, Bleyer A, Siscovick DS: Cystatin C and subclinical brain infarction. J Am Soc Nephrol 2005; 16:3721-3727. 